\title{
Food Based Dietary Guidance - Weighing up the recommendations
}

\author{
Michael Dolan ${ }^{1,2}$, Sali Abouhajar ${ }^{1,2}$, Damian O’Kelly ${ }^{2}$, Elizabeth O’Sullivan ${ }^{1}$, Aileen Kennedy ${ }^{1}$ \\ and Frances Douglas ${ }^{2}$ \\ ${ }^{1}$ Technological University Dublin, Kevin Street, Dublin 8, Ireland and \\ ${ }^{2}$ Nutritics, Nutrition Analysis Software, Town Plaza, Swords, Dublin, Ireland
}

\section{Abstract}

Food Based Dietary Guidelines (FBDG) are dietary recommendations described in terms of amounts of foods that should be consumed. They are designed to support healthy eating through simple messaging and indicate how to achieve a nutritionally adequate diet. However, assessing compliance with FBDG targets can be difficult. The quantity of food either being consumed or prescribed and the amount of that food that constitutes a serving are needed to calculate the contribution of the food towards a food group target. The aim of this study was to define the serving weight of foods, guided by definitions from Irish FBDG, to the McCance and Widdowson Composition of Foods Integrated Dataset 2015 (CoFID). This will enable the automatic assessment of compliance to FBDG using Nutritics nutrition analysis software. Foods from CoFID were categorised into six food groups as defined by Ireland's FBDG. Quantified servings from the Irish FBDG were matched to foods. Calculation criteria were developed to establish a serving size for remaining foods within each food group. For fats and oils, as well as raw fruits and vegetables, household measurements were converted to grams, using Food Portion Sizes from the Food Standards Agency. Quantities for cooked fruits and vegetables were calculated using weight-change factors published by Bognar. For the breads, cereals and potatoes group a serving size was calculated using the midpoint for the calorie bands in this group, as defined by the Food Safety Authority of Ireland. For the dairy group, a serving size was determined by calculating the quantity needed to provide $250 \mathrm{mg}$ of calcium. This was in line with achieving the total daily recommended amount of calcium from the dairy group from 3 servings. For meat, fish and alternatives (MFA), serving sizes were developed using the recommended amount of protein per body weight reference value for males and females. For foods high in fat, sugar and salt, serving sizes were defined using 100kcal as the recommended amount of energy provided for snacks. Out of 3,291 foods, 1,980 were grouped into 6 food groups. Quantified servings were available for 694 foods in 3 food groups. Calculated serving sizes were developed for the remaining 1,276 foods. The quantity of each food that constitutes a serving will be integrated into Nutritics to automatically assess compliance to FBDG. This will enable Health Care and Food Industry Professionals to deliver informed advice on meeting population health targets.

\section{Conflict of Interest}

The authors F.Douglas and D.O'Kelly provided supervision from the private company Nutritics Ltd. 